\title{
Enhanced Speed and Current Control of PMSM Drives by Perfect Tracking Algorithms
}

\author{
Koichi Sakata \\ Yokohama National University \\ Yokohama, Japan \\ Email: sakata@hfl.dnj.ynu.ac.jp
}

\author{
Hiroshi Fujimoto \\ The University of Tokyo \\ Kashiwa, Chiba, Japan \\ fujimoto@k.u-tokyo.ac.jp
}

\author{
Luca Peretti and Mauro Zigliotto \\ University of Padova \\ Vicenza, Italy \\ Email: luca.peretti@unipd.it \\ mauro.zigliotto@unipd.it
}

\begin{abstract}
Speed and current closed loops control represent the heart of any advanced AC servo drive. These inner loops usually feature high-dynamic feedback control, with possible axes decoupling and a straight feedforward action of the backelectromotive force (back-EMF). More sophisticated techniques as single-rate or multi-rate control could be exploited for both speed and current closed loops, and their performances compared to that of the classic cascade feedback controllers. This represents the goal of the present work, focusing on permanent magnet synchronous motor (PMSM) drives.
\end{abstract}

\section{INTRODUCTION}

PMSM drives for industrial applications usually feature a classic cascade structure, with an inner current control loop and an outer speed control loop. Usually, the regulators are simple PI controllers, designed as to match specific requirements such as bandwidth and phase margin.

In the recent past years, new keen control strategies and approaches emerged. Robust control using a disturbance observer [1] and adaptive control using a self-tuning regulator [2] have improved the speed control. The current control has also been improved [3]. These are all feedback approaches.

Then, two-degrees-of-freedom (2-DOF) systems which consist of not only feedback controllers but also feedforward controllers are capable of superior tracking performances with respect to classical cascade systems. One example is represented by the perfect tracking control (PTC) strategy [4], which is a well-known theory for the design of 2-DOF systems.

Performances of the PTC for the outer control loops, such as the speed loop, have been reported in previous works as [4] and [5]. Usually, since the controlled system is described by a transfer function with relative degree equal or greater than two, a multi-rate approach is needed to design a feedforward controller and guarantee perfect tracking [4]. No extended investigations are reported so far for the case of single-rate feedforward approaches, where the relative degree of the transfer function is equal to one. This is the case of the inner current control loop of a PMSM drive.

The hypothesis being presented in this work is that some of the advantages of PTC can be profitably shifted to the inner control loop, namely the current one. The combination of single-rate feedforward for the control loop and multi-rate feedforward for the speed loop can improve performances with
Table I

PMSM PARAMETERS.

\begin{tabular}{|l|c|l|}
\hline Inductance $L$ & 130 & $\mathrm{mH}$ \\
\hline Resistance $R$ & 5.15 & $\Omega$ \\
\hline Inertia $J$ & $4.0 \times 10^{-4}$ & $\mathrm{~kg} \cdot \mathrm{m}^{2}$ \\
\hline Viscosity $B$ & $3.0 \times 10^{-3}$ & $\mathrm{~kg} /(\mathrm{m} \cdot \mathrm{s})$ \\
\hline Torque coefficient $K_{t}$ & 0.44 & $\mathrm{mN} \cdot \mathrm{m} / \mathrm{A}$ \\
\hline Back-EMF constant $K_{e}$ & 0.22 & $\mathrm{~V} \cdot \mathrm{s} / \mathrm{rad}$ \\
\hline
\end{tabular}

respect of classical cascade feedback approaches. The paper gives the evidence of unabated speed and current tracking capability of the proposed approach with reduced PWM carrier frequency, with evident energy saving with respect to a highbandwidth cascade feedback controller with higher PWM carrier frequency.

The paper illustrates the mathematical passages and the needed background on PTC single-rate and multi-rate approaches, and it contains experimental results on a PMSM drive. First, the cascade current feedback control is redesigned based on pole placement theory. Then, a single-rate feedforward controller is designed for the current control. The carrier frequency is decreased for the system with the feedforward controller, showing that tracking performances are the same as the classical cascade approach with higher carrier frequency. The PMSM drive is completed with the PTC multi-rate control applied to the outer speed loop. The robustness of the PTC multi-rate feedforward controller is verified both theoretically and experimentally.

\section{IMPROVEMENTS OF THE $q$ AXIS CURRENT CONTROL}

In the first part of the work, a single-rate feedforward controller along with a classic feedback controller were applied for the $q$-axis control of a PMSM, whose data are reported in Table I. A block scheme of a 2-DOF control system composed by a single-rate feedforward controller $C_{1}[z]$ and a feedback controller $C_{2}[z]$ is reported in Fig. 1. The $S$ block represents the sample-and-hold operation where $T_{s}$ is the PWM sample time, while the presence of the delay between $r[k]$ and $y_{d}[k]$ will be cleared in the next Section II-B.

\section{A. Design of the feedback controller}

A block diagram which comprises the current $q$ axis of a PMSM and the mechanical system is shown in Fig. 2. 


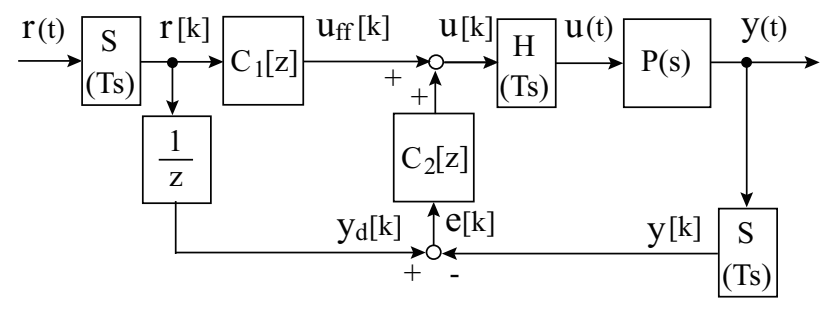

Fig. 1. 2-DOF control system in singlerate.

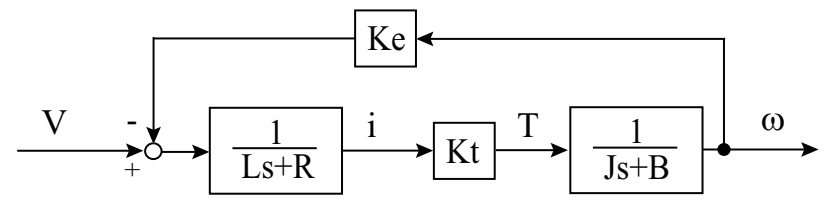

Fig. 2. Plant model of PMSM in $q$ axis.

Usually, the feedback controller is designed without taking into account the back-EMF term, so that the considered plant model for the design of $C_{2}[z]$ is:

$$
P_{i 1}(s)=\frac{i}{V}=\frac{1}{L s+R} .
$$

Here, a feedback controller is designed as

$$
C_{i 2}(s)=\frac{L s+R}{\tau_{i} s},
$$

so that the closed-loop transfer function between the reference and the measured current, neglecting the back-EMF term, is equal to

$$
\frac{i}{i^{r e f}}=\frac{1}{\tau_{i} s+1},
$$

where $\tau_{i}=1 /\left(2 \pi f_{i}\right)$. The parameter $f_{i}$ is selected as the bandwidth of the current loop. In the classic approach, the coupling term due to the back-EMF is rejected by a general decoupling control, which consists of adding to the voltage $V$ a term equal to $K_{e} \omega$.

\section{B. Design of the feedforward controller}

The feedforward component $C_{1}[z]$ of Fig. 1 was designed with a current model that considers the back-EMF contribution. In this case, the transfer function between voltage and current reported in Fig. 2 is:

$$
P_{i}(s)=\frac{i}{V}=\frac{J s+B}{L J s^{2}+(R J+L B) s+R B+K_{t} K_{e}} .
$$

The plant model $P_{i}(s)$ is discretized by a zero-order-hold (ZOH) discretization without unstable zeros, obtaining a discrete plant model named $P_{i}[z]$. The feedforward controller is then designed as:

$$
C_{i 1}[z]=\frac{1}{z P_{i}[z]}
$$

where one delay operator $z$ is needed for the feedforward controller $C_{1}[z]$ to be a biproper transfer function. Thus, when plant is nominal,

$$
y[k]=\frac{1}{z} r[k] .
$$

Here, if the reference $r[k]$ of $C_{1}[z]$ is equal to the desired output $y_{d}[k+1]$ of Fig. 1, perfect tracking is achieved as

$$
y_{d}[k]=y[k],
$$

in singlerate.

In the previous literature [6], a multi-rate feedforward controller was designed from the precise plant model including back-EMF term. However, in this case a stable single-rate feedforward controller can be designed and exploited. The reason is that a single-rate approach is not only easier, but it can also guarantee perfect tracking for a smaller sample time.

\section{Experiments}

First, the target trajectory of the current was set as a sinusoidal wave with a frequency of $100 \mathrm{~Hz}$. Two control systems consisting of only feedback controls were performed, one with a bandwidth of $1000 \mathrm{~Hz}$ and the other with a bandwidth of $100 \mathrm{~Hz}$. Here, the decoupling control to suppress the back-EMF term is employed in both control systems. Results are reported in the two upper plots of Fig. 3.

The tracking performance of the feedback control whose bandwidth is $1000 \mathrm{~Hz}$ is remarkable, while that with a $100-\mathrm{Hz}$ bandwidth is poor. A delay of 45 degrees and an attenuation of $3 \mathrm{~dB}$ are observed, as theoretically predictable because of the reference frequency of $100 \mathrm{~Hz}$. Carrier frequencies were also artificially modified, using a $10 \mathrm{kHz}$ carrier for the 1000 $\mathrm{Hz}$ bandwidth system and a $5 \mathrm{kHz}$ carrier for the $100-\mathrm{Hz}$ bandwidth system.

In the third plot of Fig. 3, a 2-DOF system which consists of the 100-Hz-bandwidth feedback controller and the single-rate feedforward controller was exploited. Carrier frequency was set to $5 \mathrm{kHz}$. Performances are better than the system without the feedforward controller, and comparable or better of those with the 1000-Hz-bandwitdh feedback controller.

The same experiment was repeated using a different current reference, that was a first-order delayed step-type trajectory, in order to test the transient response. The time constant of the trajectory is equal to $1 \mathrm{~ms}$. Results are reported in Fig. 4.

Again, the performances of the 2-DOF system with lowbandwidth feedback and feedforward are superior with respect to the low-bandwidth case. As before, they are comparable to those of the feedback controller with high bandwidth. The main advantage, however, is that the carrier frequency was halved, with evident energy saving. A bandwidth of 1000 $\mathrm{Hz}$ for the classic feedback controller cannot be practically achieved for a system with $5-\mathrm{kHz}$ carrier frequency, so performances of the classic approach would have not been the same as the ones of the feedforward approach. 

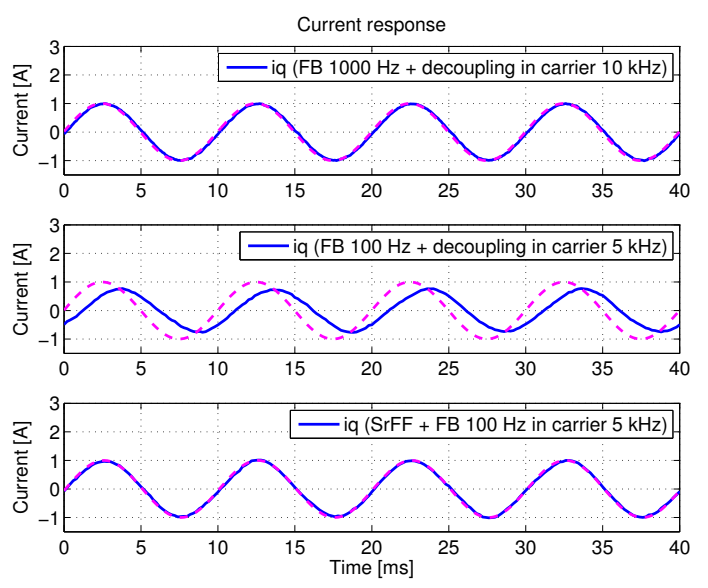

(a) Currents

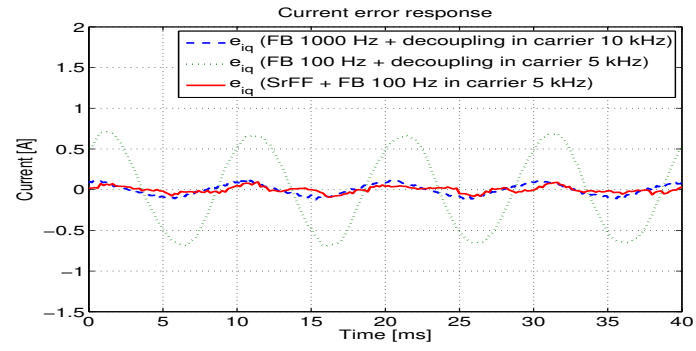

(b) Current errors

Fig. 3. Current control experimental results: sinusoidal reference.

\section{IMPROVEMENT OF THE SPEED CONTROL}

Considering again Fig. 2, the transfer function between the voltage $V$ and the mechanical speed $w$ is:

$$
P_{\omega}(s)=\frac{\omega}{V}=\frac{K_{t}}{L J s^{2}+(R J+L B) s+R B+K_{t} K_{e}} .
$$

Here, the discrete plant by $\mathrm{ZOH}$ has an almost unstable zero, because the relative degree of the transfer function is equal to two. If a single-rate feedforward controller was designed, the input would have led to vibrations and unwanted oscillations. The inverse system of the plant cannot be applied in single-rate discrete-time [7]. Therefore, the multi-rate technique is needed to design a feedforward controller for perfect tracking.

\section{A. Design of the feedback controller}

Considering a perfect tracking between the current reference and the actual current, the speed plant model is:

$$
P_{\omega 1}(s)=\frac{\omega}{i^{r e f}}=\frac{K_{t}}{J s+B} .
$$

The feedback controller is then designed as

$$
C_{\omega 2}(s)=\frac{J s+B}{K_{t} \tau_{\omega} s},
$$

so that the closed-loop transfer function between the speed reference and the actual speed is

$$
T_{\omega}(s)=\frac{\omega}{\omega^{r e f}}=\frac{1}{\tau_{\omega} s+1},
$$
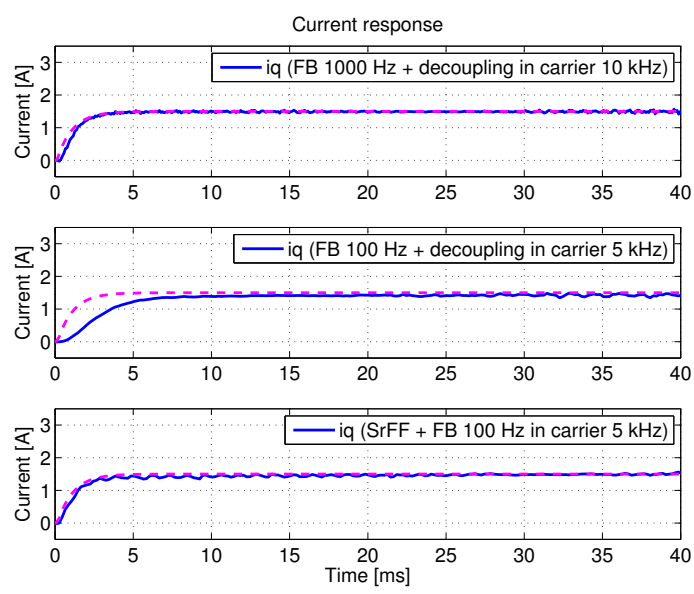

(a) Currents

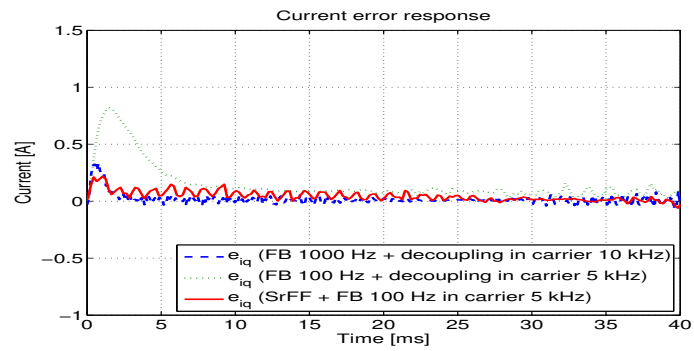

(b) Current errors

Fig. 4. Experimental results 2 of current control.

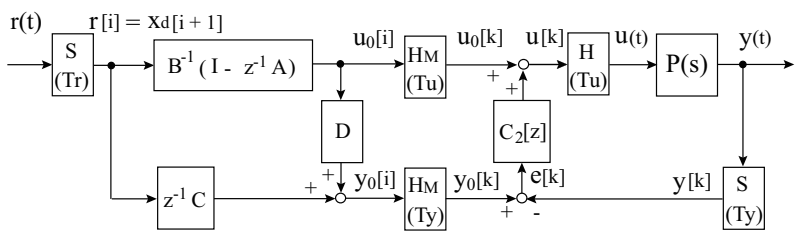

Fig. 5. Perfect tracking control system.

where $\tau_{\omega}=1 /\left(2 \pi f_{\omega}\right)$. The parameter $f_{\omega}$ is selected as the bandwidth of the speed loop.

\section{B. Perfect tracking control}

A PTC approach block diagram is reported in Fig. 5.

This system has two samplers for the reference signal $r(t)$ and the output $y(t)$, and one holder for the system input $u(t)$. Therefore, there exist three sampling periods $T_{r}, T_{y}$, and $T_{u}$ which represent the periods of $r(t), y(t)$, and $u(t)$, respectively.

PTC applies the multi-rate feedforward control in which the control input $u(t)$ is changed $n$ times during one sampling period $T_{r}$ of the reference input $r(t)$, where $n$ is the plant order. $H_{M}$ of Fig. 5 represents the multi-rate holder which outputs the input $u[i]=\left[u_{1}[k], \cdots, u_{n}[k]\right]^{T}$, generated from the long sampling period $T_{r}$ to the short sampling period $T_{u}$. Fig. 6 summarizes the concept.

From the plant model discretized by the short sampling 


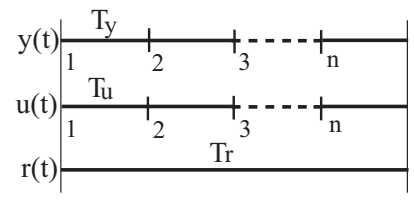

Fig. 6. Multi-rate sampling period.

period $T_{u}$, described as

$$
\boldsymbol{x}[k+1]=\boldsymbol{A}_{s} \boldsymbol{x}[k]+\boldsymbol{b}_{s} u[k], y[k]=\boldsymbol{c}_{s} \boldsymbol{x}[k],
$$

the matrices $\boldsymbol{A}, \boldsymbol{B}, \boldsymbol{C}$ and $\boldsymbol{D}$ are given as:

$$
\left[\begin{array}{c|c}
\boldsymbol{A} & \boldsymbol{B} \\
\hline \boldsymbol{C} & \boldsymbol{D}
\end{array}\right]=\left[\begin{array}{c|cccc}
\boldsymbol{A}_{s}^{n} & \boldsymbol{A}_{s}^{n-1} \boldsymbol{b}_{s} & \cdots & \boldsymbol{A}_{s} \boldsymbol{b}_{s} & \boldsymbol{b}_{s} \\
\hline \boldsymbol{c}_{s} & 0 & \cdots & 0 & 0 \\
\boldsymbol{c}_{s} \boldsymbol{A}_{s} & \boldsymbol{c}_{s} \boldsymbol{b}_{s} & \cdots & 0 & 0 \\
\vdots & \vdots & \ddots & \vdots & \vdots \\
\boldsymbol{c}_{s} \boldsymbol{A}_{s}^{n-1} & \boldsymbol{c}_{s} \boldsymbol{A}_{s}^{n-2} \boldsymbol{b}_{s} & \cdots & \boldsymbol{c}_{s} \boldsymbol{b}_{s} & 0
\end{array}\right],
$$

Since the matrix $\boldsymbol{B}$ of (13) is non-singular, PTC can be designed as

$$
\begin{aligned}
\boldsymbol{u}_{0}[i] & =\boldsymbol{B}^{-1}\left(\boldsymbol{I}-z^{-1} \boldsymbol{A}\right) \boldsymbol{x}_{d}[i+1] \\
& =\left[\begin{array}{c|c}
\mathbf{0} & \boldsymbol{I} \\
\hline-\boldsymbol{B}^{-1} \boldsymbol{A} & \boldsymbol{B}^{-1}
\end{array}\right] \boldsymbol{x}_{d}[i+1], \\
y_{0}[i] & =z^{-1} \boldsymbol{C} \boldsymbol{x}_{d}[i+1]+\boldsymbol{D} \boldsymbol{u}_{0}[i] .
\end{aligned}
$$

Expression (14) is the stable inverse system of the plant when the references are state variables $\boldsymbol{x}_{d}[k+1]$ (see Fig. 5). Therefore, the perfect tracking is assured on the sampling period $T_{r}$. Feedback control $C_{2}[z]$ suppresses the error between the output $y[k]$ and the nominal output $y_{0}[k]$ to assure robustness only when mismatch on plant parameters occurs.

\section{Control system design}

PTC is applied to a control system which consists of a cascade feedback for the current loop and the velocity loop.

The controllable canonical form of (8) is given by

$$
\begin{gathered}
\dot{\boldsymbol{x}}(t)=\boldsymbol{A}_{c} \boldsymbol{x}(t)+\boldsymbol{b}_{c} u(t), y(t)=\boldsymbol{c}_{c} \boldsymbol{x}(t), \\
{\left[\begin{array}{c|c}
\boldsymbol{A}_{c} & \boldsymbol{b}_{c} \\
\hline \boldsymbol{c}_{c} & 0
\end{array}\right]=\left[\begin{array}{cc|c}
0 & 1 & 0 \\
\frac{-\frac{R B+K_{t} K_{e}}{L \cdot J}}{-\frac{R J+L B}{L \cdot J}} & \frac{K_{t}}{L \cdot J} \\
\hline 1 & 0 & 0
\end{array}\right],}
\end{gathered}
$$

where $\boldsymbol{x}=\left[\begin{array}{ll}\omega & \dot{\omega}\end{array}\right]^{T}$. The multi-rate feedforward controller is designed by discretizing (16) with sampling period $T_{u}$, and setting $T_{u}=T_{y}=T_{r} / 2$. Matrices $\boldsymbol{A}, \boldsymbol{B}, \boldsymbol{C}$, and $\boldsymbol{D}$ are designed according to (13).

In order to obtain the the nominal current $i_{0}$ to feed the inner current feedback controller, two matrices $\boldsymbol{C}^{\prime}$ and $\boldsymbol{D}^{\prime}$ are introduced, using (13) and the output equation of the current plant model (4):

$$
y=\boldsymbol{c}_{c}^{\prime} \boldsymbol{x}, \boldsymbol{c}_{c}^{\prime}=\left[\begin{array}{cc}
\frac{B}{K_{t}} & \frac{J}{K_{t}}
\end{array}\right] .
$$

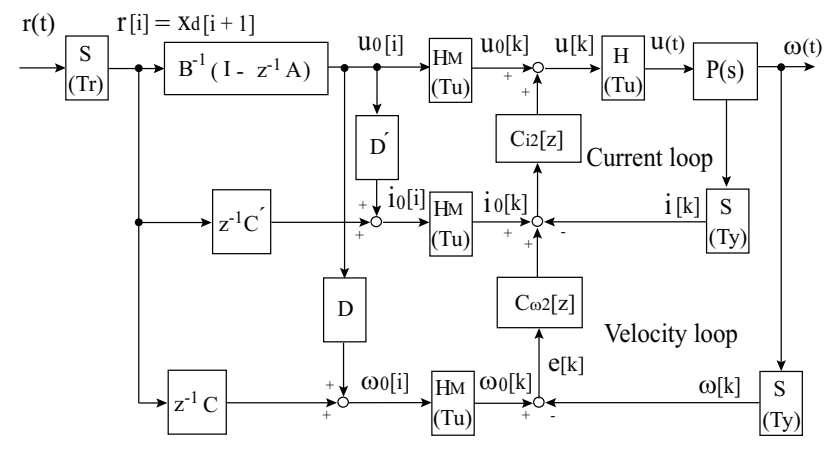

Fig. 7. Proposed control system.
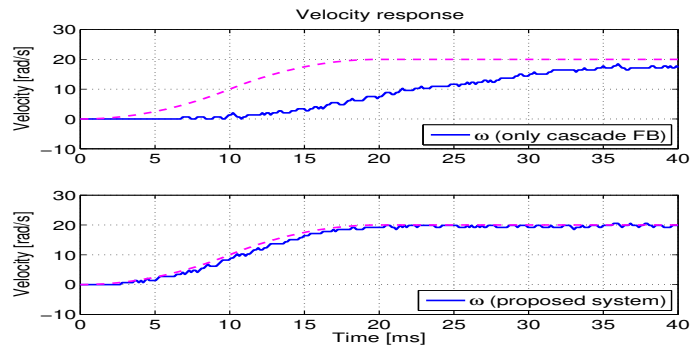

(a) Velocities

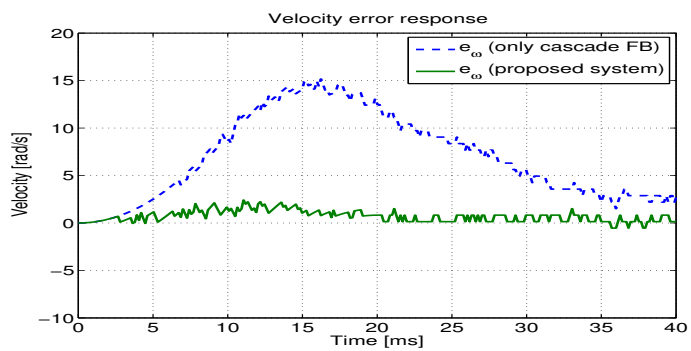

(b) Velocity errors

Fig. 8. Speed control experimental results

Fig. 7 shows the complete proposed system. Again, feedback current controller $C_{i 2}[z]$ and feedback velocity controller $C_{\omega 2}[z]$ work only when parameter mismatches occur.

\section{Experiments}

Experiments were performed in order to compare the proposed system with the conventional cascade feedback system. The target speed trajectory was set as a third-order polynomial, and carrier and control period were both set to $140 \mu \mathrm{s}$. Bandwidth of the current loop was set to $100 \mathrm{~Hz}$, while that of the speed loop was set to $10 \mathrm{~Hz}$.

Fig. 8 shows the experimental results. The proposed system shows better performances with respect to the conventional approach. In detail, two samples delay occur in order to calculate the real speed by difference between two positions. Therefore, the target trajectory of Fig. 8 and the nominal speed $\omega_{0}[k]$ of Fig. 7 are delayed by two samples. 

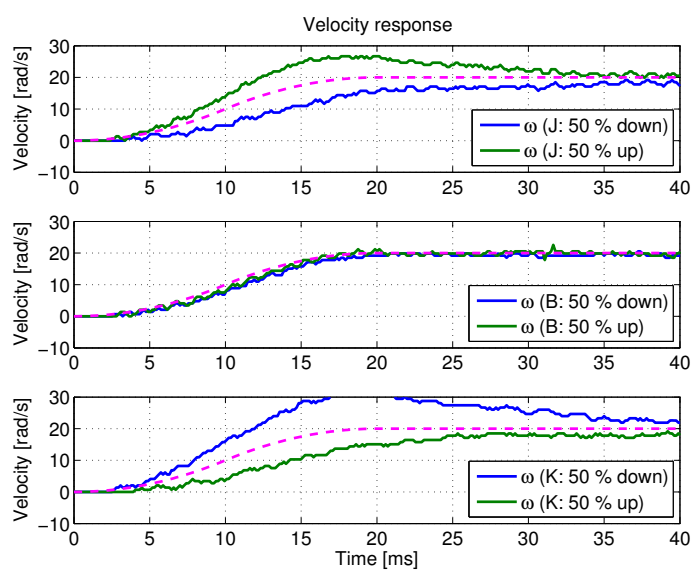

(a) Speed transients (changed $J, B$ and $K_{t}\left(=2 K_{e}\right)$ )
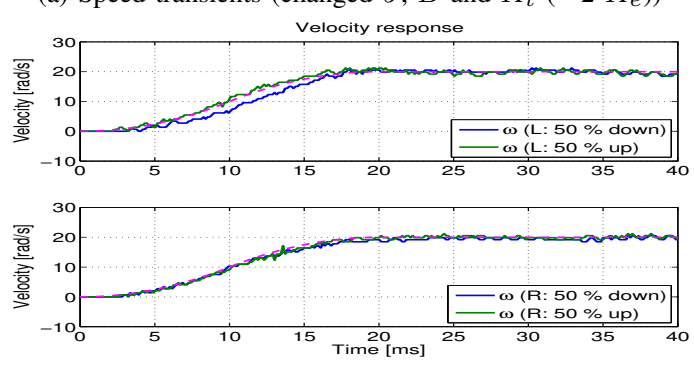

(b) Speed transients (changed $L$ and $R$ )

Fig. 9. Experimental robustness of the multi-rate feedforward control.

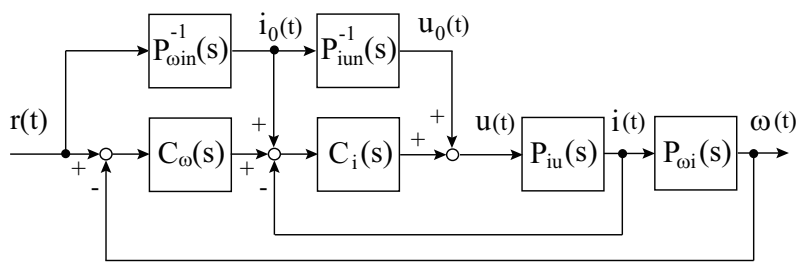

Fig. 10. Proposed control system simplified in the continuous time domain

\section{E. Robustness of the multi-rate feedforward controller}

The robustness of the multi-rate feedforward controller of PTC was experimentally verified. Each parameter used to design the multi-rate feedforward controller was changed from $-50 \%$ to $+50 \%$ of its nominal value, while the feedback controllers were not changed. Fig. 9 reports the obtained results: the most sensitive parameter is the torque constant $K_{t}\left(=2 K_{e}\right)$, while the load inertia $J$ is second one. Variation of the inductance $L$ is the third sensitive parameter, while the controller was robust against the variations of the viscosity $B$ and the resistance $R$.

\section{F. Robustness theoretical analysis}

A theoretical approach to the robustness of the proposed control system was performed. Fig. 10 shows the block scheme of the control system simplified in the continuous time domain.

$P_{i u}(s)$ from the input to the current is equal to (4) and $P_{\omega i}(s)$ is equal to (9). It is assumed that nominal stable inverse systems $P_{\text {iun }}^{-1}(s)$ and $P_{\omega i n}^{-1}(s)$ can be designed.

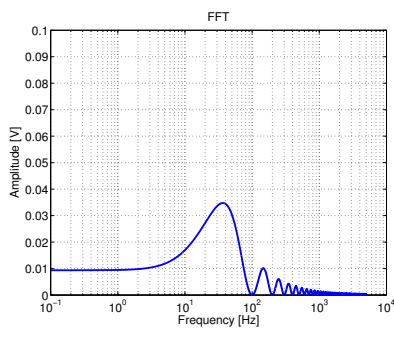

(a) $\Delta u_{0}$

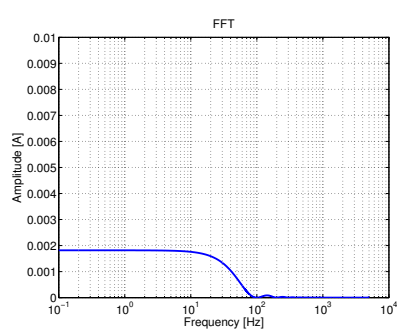

(b) $\Delta i_{0}$
Fig. 11. Bode diagram magnitude of $\Delta u_{0}$ and $\Delta i_{0}(J:+50 \%$ of nominal value).

The tracking characteristic from the reference to the output is represented as (neglecting the dependence on $s$ ):

$$
\frac{\omega}{r}=\frac{P_{\omega i} P_{i u}\left(C_{i} C_{\omega}+C_{i} P_{\omega i n}^{-1}+P_{\omega i n}^{-1} P_{i u n}^{-1}\right)}{P_{\omega i} P_{i u} C_{i} C_{\omega}+P_{i u} C_{i}+1} .
$$

If the plant is nominal $\left(P_{i u}=P_{i u n}\right.$ and $\left.P_{\omega i}=P_{\omega i n}\right)$, perfect tracking is achieved $(\omega(t)=r(t))$. When parameter mismatches exist, parts of the feedforward inputs $u_{0}$ and $i_{0}$ are to be considered as disturbances. The input variations are defined as

$$
\begin{aligned}
\Delta i_{0} & =\left(P_{\omega i}^{-1}(s)-P_{\omega i n}^{-1}(s)\right) r \\
\Delta u_{0} & =\left(P_{\omega i}^{-1}(s) P_{i u}^{-1}(s)-P_{\omega i n}^{-1}(s) P_{i u n}^{-1}(s)\right) r .
\end{aligned}
$$

In case of an inertia $J$ variation of $+50 \%$ with respect of its nominal value, the magnitude of the Bode diagrams of the variations (19) are reported in Fig. 11. Speed control and current control bandwidths have been set to $10 \mathrm{~Hz}$ and 100 $\mathrm{Hz}$, respectively.

The transfer functions between the input variations to the speed are

$$
\begin{aligned}
\frac{\omega}{\Delta i_{0}} & =\frac{P_{\omega i}(s) P_{i u}(s) C_{i}(s)}{P_{\omega i}(s) P_{i u}(s) C_{i}(s) C_{\omega}(s)+P_{i u}(s) C_{i}(s)+1} \\
\frac{\omega}{\Delta u_{0}} & =\frac{P_{\omega i}(s) P_{i u}(s)}{P_{\omega i}(s) P_{i u}(s) C_{i}(s) C_{\omega}(s)+P_{i u}(s) C_{i}(s)+1}
\end{aligned}
$$

The Bode diagram magnitudes of the (22) are shown in Fig. 12 (a). The disturbance suppression could be better in plantpole cancellation feedback control: this is due to the fact that the plant has a low mechanical pole $(-J / B)$.

In order to improve robustness, the speed PI controller $C_{\omega 2}(s)$ of (10) is redesigned without plant-pole cancellation as in (9), using the following expressions:

$$
\begin{gathered}
C_{\omega 2}(s)=K_{p}+\frac{K_{i}}{s}, \\
K_{p}=\frac{2 \zeta_{c l} \omega_{c l} J-B}{K_{t}}, K_{i}=\frac{J \omega_{c l}^{2}}{K_{t}} .
\end{gathered}
$$

With this choice, the closed-loop characteristic polynomial of the speed loop is given by

$$
A_{c l}(s)=s^{2}+2 \zeta_{c l} \omega_{c l} s+\omega_{c l}^{2} .
$$




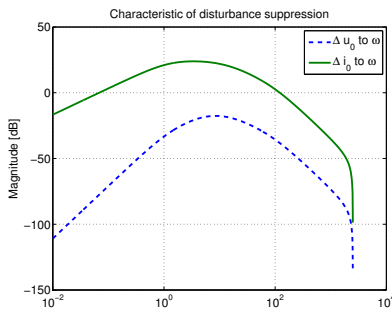

(a) with plant-pole cancellation

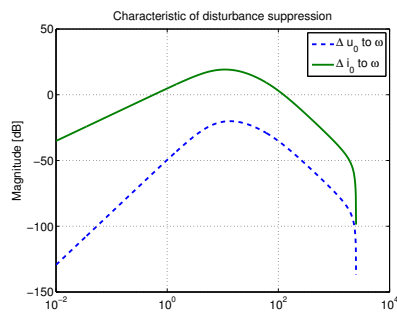

(b) w/o plant-pole cancellation
Fig. 12. Disturbance suppression transfer functions.

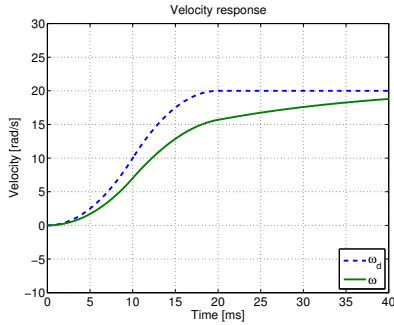

(a) With plant-pole cancellation

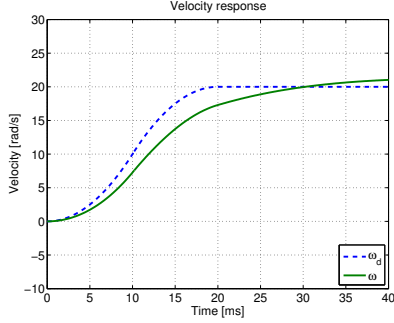

(b) Without plant-pole cancellation
Fig. 13. Time responses ( $J:+50 \%$ of nominal value).

where the damping factor $\zeta_{c l}$ was set to 1 , and $\omega_{c l}=2 \pi f_{\omega}$ where $f_{\omega}$ is the bandwidth of the speed loop. The disturbance suppression responses without plant-pole cancellation are shown in Fig. 12 (b).

The disturbance suppression is better than the one with plant-pole cancellation. This is also proved by the comparison the time responses (Fig. 13).

Finally, although stability margin is worse as shown in Fig. 14 and 15, the feedback without plant-pole cancellation still has enough stability margin in the case of an inertia variation of $+50 \%$ with respect to its nominal value.

\section{CONCLUSiON}

A single-rate feedforward control has been designed, along with a classic feedback control, in order to achieve perfect tracking for the inner current control loop of a PMSM drive. Results shows that in case of single-rate feedforward control the carrier frequency could be decreased to obtain the same performances of conventional cascade feedback approaches, with evident energy savings.

The speed loop was coupled with a multi-rate feedforward controllers were designed. Tracking performances were improved dramatically with respect to the conventional approach. Robustness of the multi-rate control against parameter variations was tested experimentally, and some hints on the theoretical approach to the robustness analysis were provided.

\section{REFERENCES}

[1] T. Umeno and Y. Hori, "Robust speed control of DC servomotors using modern two degrees-of-freedom controller design," IEEE Trans. Ind. Electron., vol. 38, no. 5, pp. 363-368, Oct. 1991.

[2] T.-J. Kweon and D.-S. Hyun, "High-performance speed control of electric machine using low-precision shaft encoder," IEEE Trans. power Electron., vol. 14, no. 5, pp. 838-849, Sep. 1999.

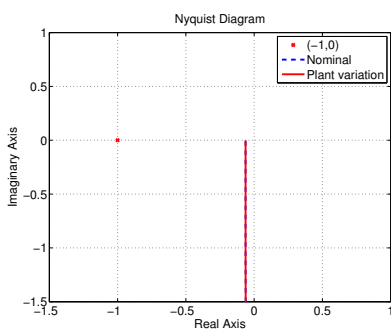

(a) Current loop

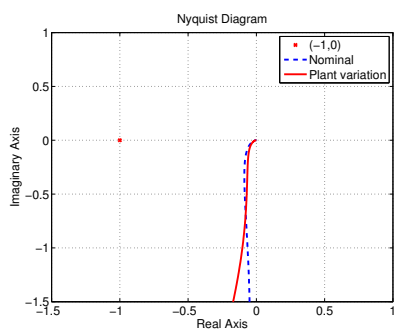

(b) Velocity loop
Fig. 14. Nyquist diagrams (with plant-pole cancellation).

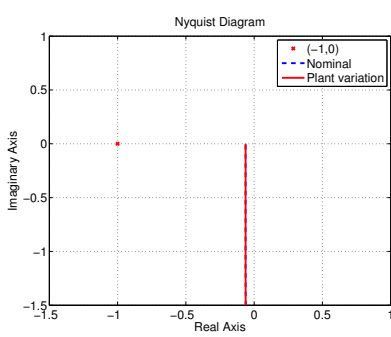

(a) Current loop

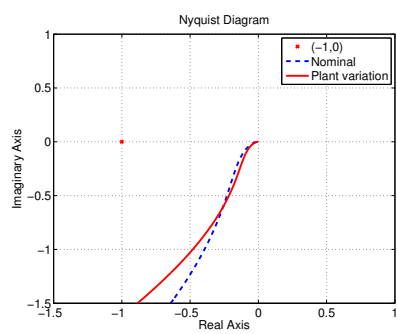

(b) Velocity loop
Fig. 15. Nyquist diagrams (without plant-pole cancellation).

[3] Y. A.-R. I. Mohamed, "Design and implementation of a robust currentcontrol scheme for a PMSM vector drive with a simple adaptive disturbance observer," IEEE Trans. Ind. Electron., vol. 54, no. 4, pp. 1981-1988, Aug. 2007.

[4] H. Fujimoto, Y. Hori, and A. Kawamura, "Perfect tracking control based on multirate feedforward control with generalized sampling periods," IEEE Trans. Ind. Electron., vol. 48, no. 3, pp. 636-644, Jun. 2001.

[5] K. Saiki, A. Hara, K. Sakata, and H. Fujimoto, "A study on high-speed and high-precision tracking control of large-scale stage using perfect tracking control method based on multirate feedforward control," in Proc. the 10th International Workshop on Advanced Motion Control, pp. 206-211, 2008.

[6] Y. Terada, T. Nakai, and H. Fujimoto, "Proposal of high-speed and high-precision control method for SPMSM based on perfect tracking control with multirate PWM-low-carrier current control and inspection of position control with high-resolution encoder-", in Proc. IEEJ-IIC, IIC-08-46, pp. 65-70, 2008 (in Japanese).

[7] K. J. Åström, P. Hangander, and J. Sternby, "Zeros of sampled system," Automatica, vol.20, no.1, pp. 31-38, 1984 